\title{
Formalization of discrete processes of forming porous materials using information models
}

\author{
Vladimir Kulikov ${ }^{1, *}$ \\ ${ }^{1}$ Moscow State University of Civil Engineering, Yaroslavskoeshosse, 26, Moscow, 129337, Russia
}

\begin{abstract}
The author has developed an information model of the material based on the analysis of their corresponding textures. The model represents the attribute information on the system state in discrete time of formation of the structure of the material. The model allows us to create an information replica of the media (texture) on the state of the system at a specific point in time and multiply it in the form of information copies of the system state on objects created in other places, in other conditions and at other time periods.
\end{abstract}

\section{Introduction}

Important functional and performance properties of various materials are determined by the peculiarities of the structure of their pores. Control of formation of porous materials allows to obtain structures with an optimal set of quality indicators. The formation of porous structure is the result of observance of technological rules. Technology of formation of structure can be formalized in a consistent heterogeneous system transitions from initial state to final state. Each such state represents a specific information base about the structure of a material at a given point in time. Development of information model for each discrete state of the system will allow to monitor and control the process of formation of highquality porous structure of the materials [1-5].

\section{How was the study done}

It is known that in closed systems, which exchange matter and energy with the environment, after some time equilibrium is established. For example Figure 1. If you combine the two parts of the vessel filled with two different gases, then over time these two gases are mixed in each other. The left part of Figure 1 contains separate information about the two parts of the same vessel. The right part of Figure 2 concentrates, balances the previous information in a single volume of the contents through the mixing process.

${ }^{*}$ Corresponding author: 9646379164@mail.ru 


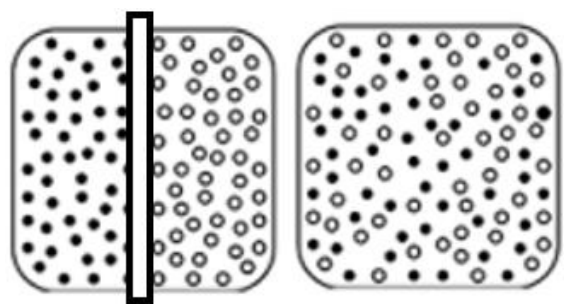

Fig. 1. Illustration reduce the amount of information about the object when it is mixed by its concentration on a single object.

As it follows from Figure 1 the concepts about the individual elements of the system remains at the same level thanks to their stirring. But the total amount of information content of concepts is reduced from 2 to 1 .

The process of making porous structures also consists of two major stages. Preparation of aerated mixture and obtaining, ultimately, a porous artificial stone. These two stages have consistently held the next state. The first - mixing. Second - gasification. The third hydration. The sequence of these transformations from left to right shown in Figure 2. Another schematically shown, and the successive phase transformations of heterogeneous system in the process of structure formation.

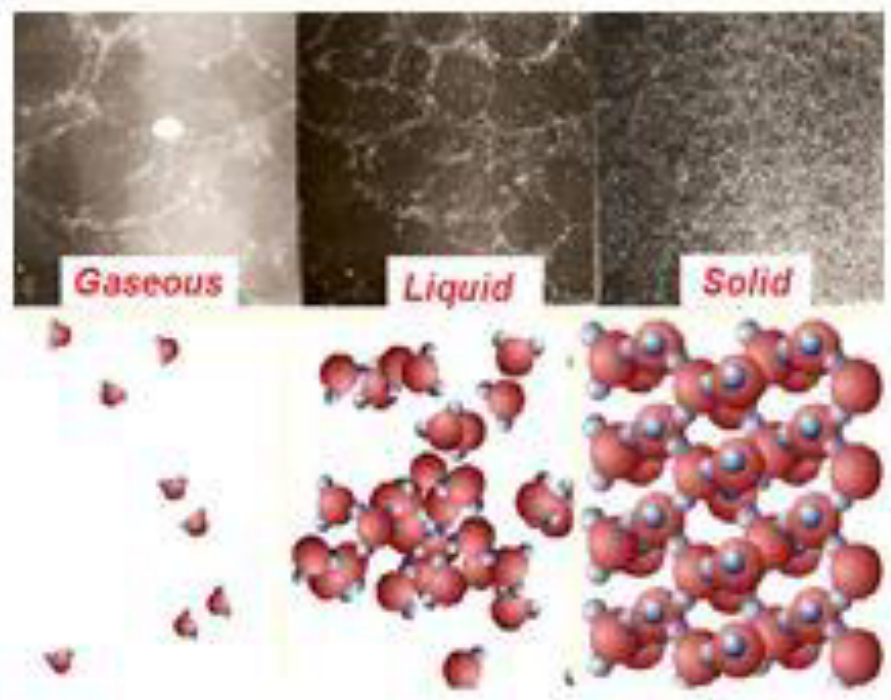

Fig. 2. Fixed on the photo, the process from left to right of the formation pore structures.

Obvious some overlap of right-hand parts of Figures 1 and 2. The right part of Figure 2 corresponds to a more balanced system than the left drawings. Thus, on the basis of the foregoing, the right picture is informative and may serve as a basis for development on its basis of information model the system state in a given time.

What are we looking for? So, source of information about the discrete state of the system can be a photo in this time.

For examining and removing information casts fixed by means of pictures stages the state of the system will use the optical editor Image J. It can measure distances and angles. It can create density histograms and line profile plots. It supports standard image processing functions such as contrast manipulation, sharpening, smoothing, edge detection and median filtering. It does geometric transformations such as scaling, rotation and flips. Image can be 
zoomed up to $32: 1$ and down to 1:32. All analysis and processing functions are available at any magnification factor.

Digital images are two-dimensional grids of pixel intensities values with the width and height of the image being defined by the number of pixels in X (rows) and Y (columns) direction. Thus, pixels (picture elements) are the smallest single components of images, holding numeric values - pixel intensities - that range between black and white.

If you build a sequence of photos from the initial state to the final, and convert them into analytical relationships, then such analytic dependencies can be adequately formalized the technology used in the production process. Consistent reproduction of these dependencies, with constant monitoring of their compliance with the original samples may allow to obtain high-quality clonesof the original samples.

\section{Results}

Consider the algorithm for obtaining information model of the system state on the basis of studying photographs of the state. In the first stage will do the following: -submitted photo converted to binary code and process it using Image J, as shown in Figure 3.
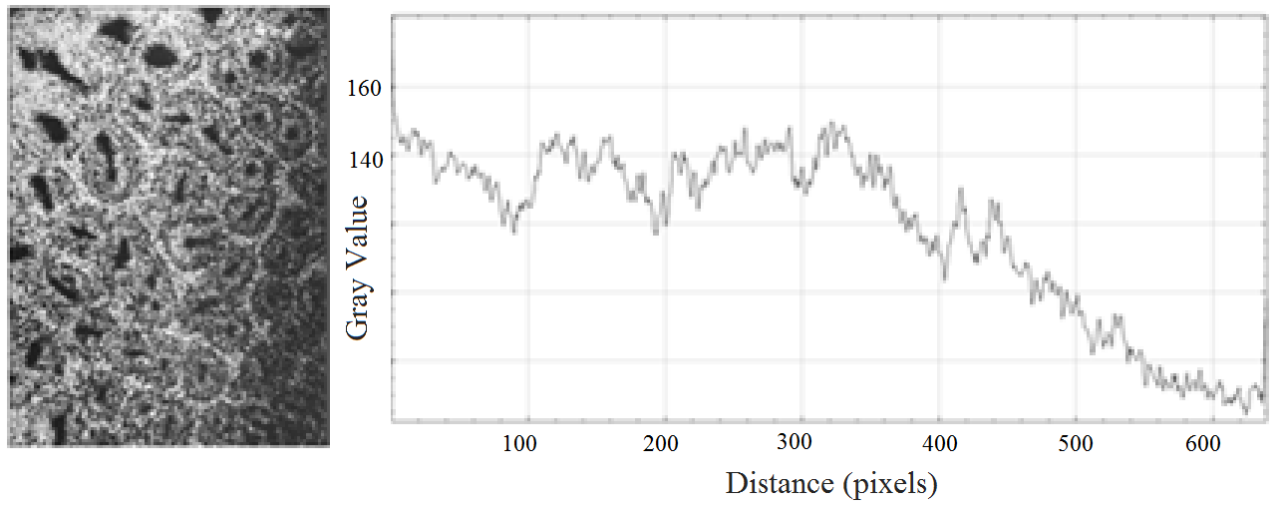

Fig. 3. Photo of the discrete state of the system is converted into a binary code and ready to be processed with Image J.

Based on the pictures in Figure 3, create its graph profile. Analysis of the data indicates the developed surface of the object. This is evidenced by the many lance-like shape along the whole length of this graph.

Information received and its amount, the results of statistical samples, their processing and the results are shown in Figure 4.

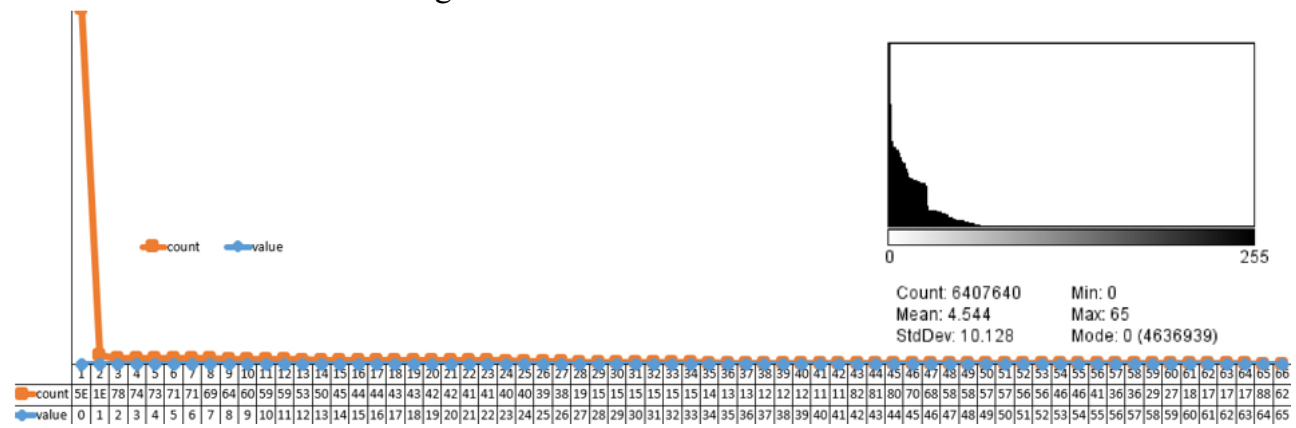

Fig. 4. The information block data to develop a model of the system state. 
The histogram of the values of the sizes of the elementary objects represented in the photograph, their variation and deviation from the mean, we show in Fig.4.

In statistics, the standard deviation is a measure that is used to quantify the amount of variation or dispersion of a set of data values. In our case, the standard deviation is 10.128 of 6407640 studied and treated elementary objects.

The expected value of a discrete set of numbers (Mean) is 4.544 for the dataset shown in Figure 4.

In fact, the block of information presented in an integrated form in Figure 4 may be a basis for finding analytical dependences between geometric shapes and their sizes. Search analytical dependences on the basis of the data presented will carry out approximation of the function $\mathrm{f}(\mathrm{x})$ such a function $\mathrm{g}(\mathrm{x})$ (approximating function), which would be close to the specified value.

The regress function is auxiliary, it prepares the data required for the function interp.

Vector s contain, in particular, the coefficients of the polynomial

The continued processing of discrete data to retrieve information model shown in Figure 5. Finding the approximating function $\mathrm{g}(\mathrm{x})$, i.e., the interpolation polynomial, we can compute the function values $\mathrm{f}(\mathrm{x})$ between nodes $\mathrm{Y}$, discrete information points, defining the concept of developed area, and to determine the value of the function $f(x)$ even outside a specific range.

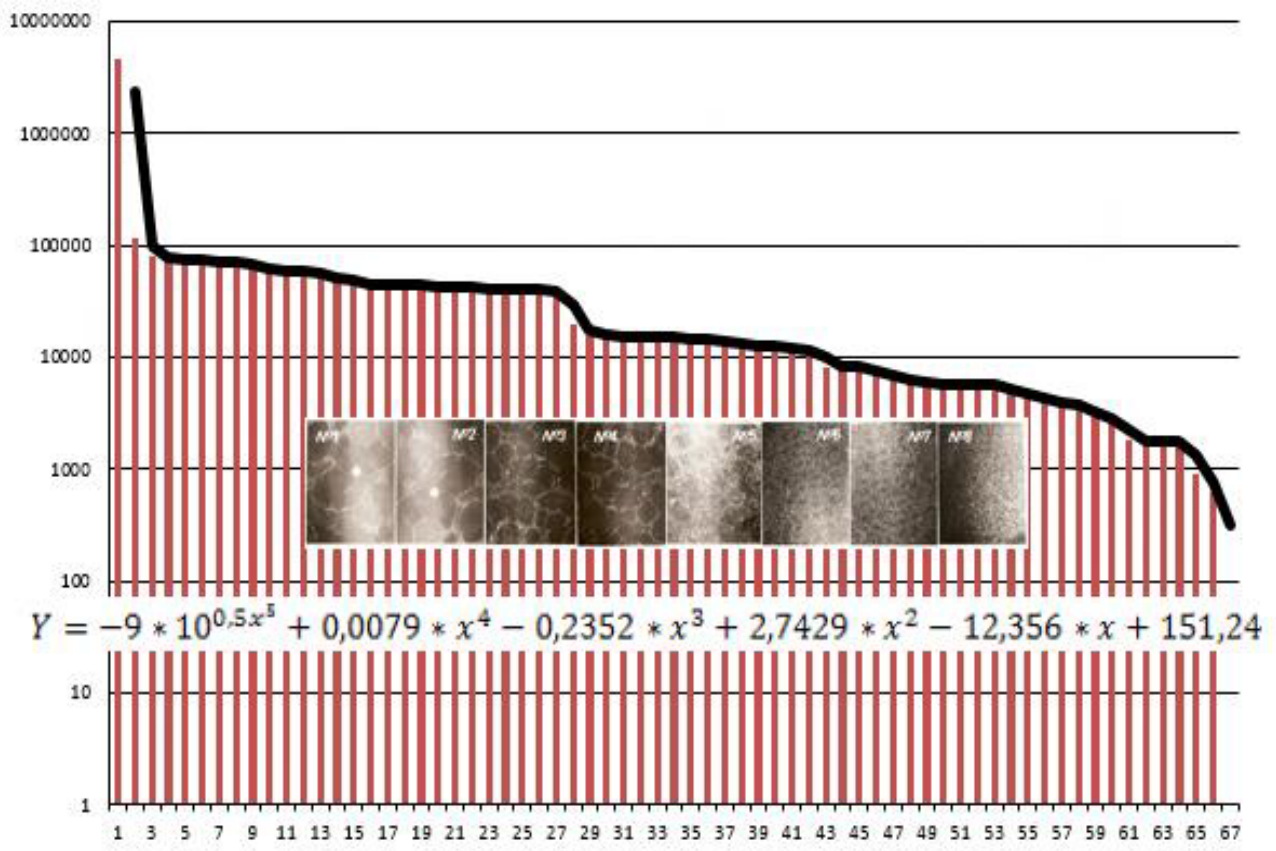

Fig. 5. The implementation of approximating functions $\mathrm{g}(\mathrm{x})$ as a polynomial of the 5 th degree.

As a function $\mathrm{g}(\mathrm{x})$ we choose the polynomial, which we call interpolation polynomial.

$$
Y=-9 \cdot 10^{0.5 x^{5}}+0.0079 \cdot x^{4}-0.2352 \cdot x^{3}+2.7429 \cdot x^{2}-12.356 \cdot x+151.24
$$

Obviously a coincidence of shapes of the graph of the surface profile in Figure 2 and obtained the analytical form of the approximation in Figure 6.

This means that the task of developing an information model of the system state we did. 


\section{Discussion}

Using photos can be discretely in time to capture all the stages of formation of the pore structures and put them in series, following each other in time, as shown in Figure 6. This sequence of photos is an informational cast of successive state changes in time due to the use of specific production technologies of porous structures. Therefore this sequence of photos may be adopted for identifying the standard for such technology. Each individual of more photos can be supplied separate information model status. As it follows from Figure 6 , these models will be 8 . Thus the production technology (T) can be formalized with the appropriate integration of information models:

$$
\mathrm{T}=\mathrm{N} 1+\mathrm{N} 2+\ldots+\mathrm{Nn}
$$

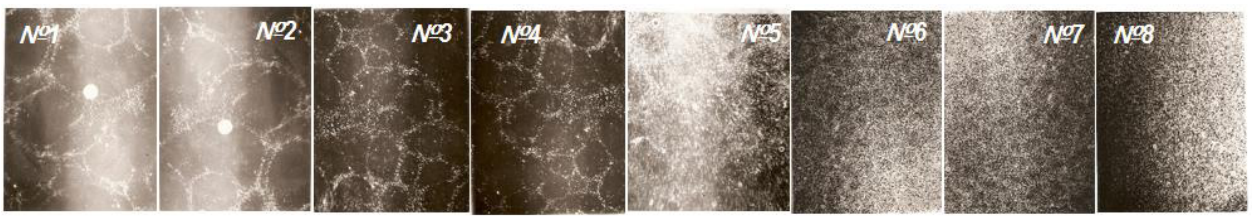

Fig. 6. Visual-information representation of the production technology of porous structures.

In our case, in the scope of this article, we showed a way of formalizing only one technological conversion, 7. But practically using the relation [2] can carry out analytical formalization of any technology.

What next? The information model of the system state in a given time [1] in the form $\mathrm{Y}=\mathrm{f}\left(\mathrm{x}_{\mathrm{i}}\right)$ can be considered flat or one-factor models. Controlling the behavior of such models can be realized by varying the value of only one factor $\left(\mathrm{X}_{\mathrm{I}}\right)$. But sophisticated technology provide the opportunity to manipulate large number of source of information criteria in the form of the factors causing the relevant technology. The work implemented a method of constructing a two-factor model in the form $Z=(x, y)$, the result is shown in Figure 8.

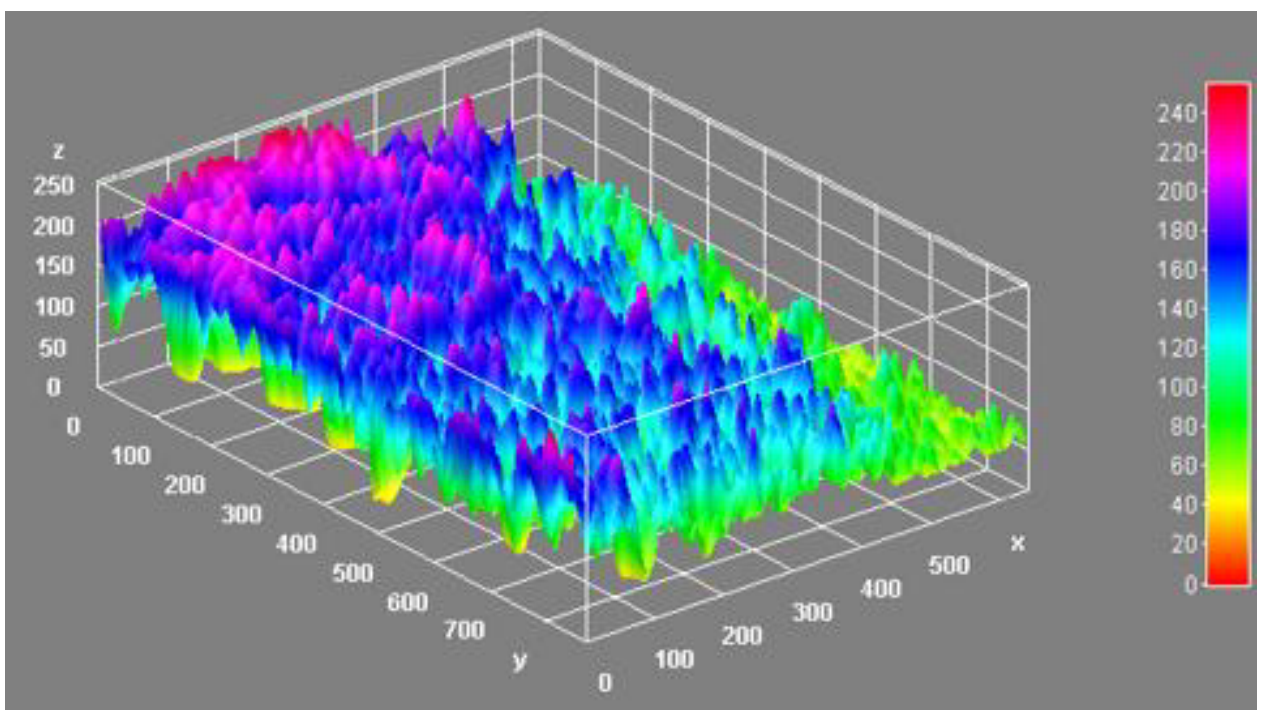

Fig. 7. Two-factor model of condition 7 from Figure 6.

Using the two-factor model can provide and assess the degree of heterogeneity of the structure of the studied material. It can help a more detailed assessment of the mutual 
position of each relative to the other clusters of pores and voids. By varying the relevant parameters of the information model to simulate the equilibrium and non equilibrium structure of the model and then, based on adjusted models to implement them in nature.

Having developed the levers of control information model, it can help guide the development of porosity in one direction or the other, to form closed or open nature of the porosity developed porous structures.

\section{Conclusion}

The aim of this work was to show the possibility of the formalization of the process of production of porous structures by means of information models. Each technological operation is submitted to the relevant information cast, - formalized the corresponding analytical dependence, for example [1]. Thus, the goal set in the work-achieved. Developed two-factor model the state of the investigated system which allows a rational way to choose the ratio of often conflicting each other two factors (criteria) technology.

\section{References}

[1] J. L. Fiadeiro, A. Lopes, Theoretical Computer Science, 663, 1-33 (2017)

[2] F. Nian, W. Liu, Solitons \& Fractals, 91, 554-561 (2016)

[3] Y. Wang, G. Chen, Optik - International Journal for Light and Electron Optics, 132, 111120 (2017)

[4] G. Caruso, D. V. Di, Maio International Journal of Heat and Mass Transfer, 68, 401-414 (2014)

[5] Q. Zhang, X. Duan, Sh. Ma, Statistics \& Probability Letters, 122, 11-19 (2017) 